Revue d'histoire de l'Amérique française

RAS REVUE D.HISTOIRE DE L'AMÉRIQUE FRANÇAISE

\title{
Économie et histoire : les liaisons dangereuses d'Hermès et de Clio
}

\section{Gilles Paquet}

Volume 46, numéro 4, printemps 1993

URI : https://id.erudit.org/iderudit/305148ar

DOI : https://doi.org/10.7202/305148ar

Aller au sommaire du numéro

Éditeur(s)

Institut d'histoire de l'Amérique française

\section{ISSN}

0035-2357 (imprimé)

1492-1383 (numérique)

Découvrir la revue

Citer cet article

Paquet, G. (1993). Économie et histoire : les liaisons dangereuses d'Hermès et de Clio. Revue d'histoire de l'Amérique française, 46(4), 629-643.

https://doi.org/10.7202/305148ar d'utilisation que vous pouvez consulter en ligne.

https://apropos.erudit.org/fr/usagers/politique-dutilisation/ 


\title{
ÉCONOMIE ET HISTOIRE: LES LIAISONS DANGEREUSES D'HERMÈS ET DE CLIO ${ }^{1}$
}

\author{
GILLES PAQUET \\ Faculté d'administration \\ Université d'Ottawa \\ "Un rationaliste sans problématique, \\ c'est une raison qui ne peut pas respirer; \\ il étouffe, il tombe dans le dogmatisme».
}

Gaston Bachelard

\section{1 - INTRODUCTION}

Quand l'Institut d'histoire de l'Amérique française m'a invité à prononcer cette conférence à une session de son congrès de l'automne 1991 qui s'intitulerait «Histoire et économie: un mariage difficile», il m'a semblé que ce titre portait à faux. La liaison de Clio et Hermès dure depuis un bon moment, elle a eu ses hauts et ses bas, mais quels que soient les vœux de la parenté, il n'a jamais été question de mariage. C'est un couple qui est condamné à vieillir dans l'illégitimité.

Personne mieux que mon maître Albert Faucher n'a su mieux cerner le statut trouble de cette relation quand il a caractérisé ses rejetons (historien économiste ou économiste historien) d'espèce hybride exposée à l'opprobre ${ }^{2}$.

1. Texte revisé d'une conférence à la session d'ouverture du congrès de l'Institut d'histoire de l'Amérique française qui se tenait à Québec les 24, 25 et 26 octobre 1991. L'assistance de Marie Saumure et les commentaires des évaluateurs anonymes ont aidé à améliorer le texte.

2. Albert Faucher, «L'histoire économique dans la province de Québec jusqu'à la fin du $\mathrm{XIX}^{\mathrm{e}}$ siècle», Recherches sociographiques, 3,1-2 (1962). 
Cette liaison houleuse a pourtant une histoire intéressante qui pourrait même être en train de prendre un tour nouveau. C'est pourquoi il n'est pas inutile peut-être de faire le point, question de prendre la mesure des futurs possibles à l'aune des expériences passées.

Dans un premier temps, les misères de cette relation au cours des dernières décennies sont exposées. Ces difficultés, on peut les expliquer par des incompatibilités de caractères qui semblent incontournables. Cependant pas question de nier que Clio et Hermès ont été entrevus dans certains sous-bois récemment et que ces fréquentations nouvelles semblent prometteuses pour certains. Notre conclusion sera plus nuancée car il faut bien comprendre que la belle est volage et que le beau a pris beaucoup de mauvaises habitudes, et que ces retrouvailles dans le monde interlope des écoles de gestion ou d'ingénierie ne promettent pas nécessairement une liaison extramaritale durable.

\section{2 - UN PEU D'HISTOIRE}

L'histoire économique traditionnelle (i.e. l'histoire des faits économiques) a une longue tradition au Canada et au Québec: les travaux d'Adam Shortt et d'Errol Bouchette en témoignent. Mais la première grande relation, le coup de foudre qui va conduire à une relation perfecte intime omne entre Hermès et Clio, remonte aux années 1920 et 1930 avec les travaux de W. A. Mackintosh et de Harold A. Innis. Ce fut d'abord un gambit d'Hermès: les économistes venant sur le terrain des historiens. Mais Clio devait répondre assez spontanément à ces avances: ce sont les travaux d'A. R. M. Lower et de D. G. Creighton. De ces fréquentations va sortir l'histoire économique moderne au Canada ${ }^{3}$.

Ce passage à une liaison durable va se faire en trois mouvements.

Le premier est le développement d'un outillage économique qui permette d'interpréter la réalité canadienne. Hermès va ambitionner de

3. Pour un survol des travaux en science économique au Canada et au Québec, voir Robin Neill, A History of Canadian Economic Thought (London, 1991), et Gilles Paquet, La pensée économique au Québec français (Montréal, 1989). Pour des essais bibliographiques sur l'histoire économique au Québec et au Canada, voir Gilles Paquet et Jean-Pierre Wallot, «Canada 1760-1850: anamorphoses et prospective», R. Comeau, dir., Économie québécoise (Montréal, 1969), 255-300; «Pour une méso-histoire du XIX ${ }^{e}$ siècle canadien», Revue d'histoire de l'Amérique française, 33,3 (décembre 1979): 387-425. Certains travaux illustrent bien la contribution des économistes et historiens mentionnés ici en passant pour illustrer notre propos: W. A. Mackintosh, «Economic Factors in Canadian History», Canadian Historical Review, 4 (1923): 12-25; Harold A. Innis, The Problems of Staple Production in Canada (Toronto, 1933); Essays in Canadian Economic History (Toronto, 1956); Arthur R. M. Lower, Settlement and the Forest Frontier in Eastern Canada (Toronto, 1936); Donald Creighton, Commercial Empire of the St.Lawrence 1760-1850 ('Toronto, 1937). 
subjuguer Clio: c'est la fameuse théorie des «staples» ou produits générateurs qui va permettre à Innis de découper l'histoire du pays en une série de périodes chacune marquée par un produit-clé (fourrures, bois, blé, etc.).

Dans le second, Hermès va prétendre étendre son imperium à tous les proches de Clio. C'est un effort pour construire un lien clair et net entre la modélisation économique et une explication de la trame sociopolitique: une certaine arrogance d'Hermès l'amène à se déclarer capable d'interpréter praxis et mentalités à partir des mécanismes économiques. En ce sens, la nouvelle théorie économique d'Innis est fort ambitieuse.

Le troisième mouvement est celui de la quantification. Hermès va imposer à Clio une certaine métrification. On va commencer à mettre en place des séries chronologiques qui vont permettre de jauger l'évolution de certaines grandes variables économiques repérées par les analyses. C'est la mise en place des Statistical Contributions to Canadian Economic History ${ }^{4}$.

Ces efforts se font surtout au Canada anglais. Au Québec, Hermès et Clio se courtisent un peu, et Clio est fort active, mais il y a peu d'histoire économique moderne au sens où nous l'avons définie si ce n'est dans certaines pages de L'Actualité Économique.

Dans l'après Seconde Guerre mondiale, c'est-à-dire les années 1950 et 1960 , Hermès et Clio vivent un moment d'amour-passion. L'histoire économique moderne s'installe. Ce n'est plus simplement Clio poursuivie par Hermès, Clio a pris goût au jeu et poursuit maintenant Hermès avec autant d'assiduité. Jusque-là, la priorité avait été donnée au matériau historique, et l'outillage économique avait été développé pour investir ce matériau. Dans cette troisième phase du développement de l'histoire économique au Canada, les deux partenaires s'en donnent à cœur joie: il y a dialogue extraordinairement fructueux entre une science économique en pleine révolution et une histoire en train de se renouveler.

Dans toutes les directions, on voit des expérimentations. Ce sont les fresques théoriques des disciples d'Innis d'abord, John Dales et Mel Watkins et de tout le groupe de Toronto autour de Tom Easterbrook, ceux de Ken Buckley et du groupe de la Saskatchewan aussi ${ }^{5}$. Au Québec, ce sont les travaux d'Albert Faucher et d'Alfred

4. W. A. Mackintosh, C. A. Curtis, K. W Taylor, H. Mitchell, Statistical Contributions to Canadian Economic History (Toronto, 1931), vol. 1 et 2.

5. John H. Dales, «Fuel, Power and Industrial Development in Central Canada», American Economic Review - Papers and Proceedings (May 1953); Mel H. Watkins, «A Staple Theory of Economic Growth», Canadian Journal of Economics and Political Science, 29 (1963): 
Dubuc faisant chacun à leur manière une théorie de la socio-économie. Côté histoire, le Québec est en avance: c'est l'arrivée chez nous de «l'histoire sérielle» et des théorisations socio-économiques des Annales via Jean Hamelin et Fernand Ouellet surtout ${ }^{6}$.

En général, dans ce dialogue au pair, c'est Clio qui mène le bal. Hermès l'accompagne, la fait tourner, la fait rire, mais elle demeure le grand centre d'intérêt. Mais déjà on commence à voir poindre un certain machisme (dirait-on): dans les travaux de Buckley, les longs cycles mesurés ont l'air de vouloir défalquer la réalité plutôt que de lui donner du relief. Dans certains travaux d'histoire sérielle, la série mène la danse au point d'empêcher d'écouter la musique. Des économistes vont même commencer à parler de l'histoire comme d'un laboratoire pour tester leurs théories.

Quatre grandes fièvres vont alors déclencher des années orageuses dans la vie du couple Hermès-Clio et les amener même à vivre séparés pendant un moment. Rien qui semble grave au début, mais le mal va s'aggraver sérieusement dans les années 1970.

Côté Hermès, c'est d'abord une fixation des travaux des économistes sur le court terme, sur les problèmes de stabilisation, qui les amène à se détourner des problèmes de changements institutionnels dans le temps long. Il va y avoir de moins en moins d'intérêt pour l'histoire en économie.

Ensuite, et plus dramatiquement, la science économique s'est faite non seulement myope et amnésique, mais elle a pris un tour formaliste, elle s'est donné des volontés de scientifisation qui vont la faire sombrer dans un mal sournois, le méthodisme. Ce genre de virus a l'heur de dessécher dramatiquement les analyses économiques: la science économique se détache non seulement de l'étude du passé mais aussi de l'étude du présent. Les économistes commencent à fonctionner à vide: même le Nobel d'économie Leontieff pourra se plaindre au début des années 1980 que le gros des articles publiés par les économistes en Amérique sont des examens de puzzles sans rapport avec la réalité. La démarche économique devient solipsiste, onaniste diront les méchantes langues ${ }^{7}$.

141-158; W. T. Easterbrook, North American Patterns of Growth and Development (Toronto, 1990); Kenneth Buckley, Working Paper on Population, Labour Force and Economic Growth (Banff, 1963).

6. Albert Faucher, Québec en Amérique (Montréal, 1973); Alfred Dubuc, «Une interprétation économique de la constitution», Socialisme, 66 (janvier 1966): 3-21; «Les classes sociales au Canada», Annales ESC, 4 (juillet-août 1967): 829-844; Jean Hamelin, Économie et société en Nouvelle-France (Québec, 1960); Fernand Ouellet, Histoire économique et sociale du Québec 1760-1850 (Montréal, 1966).

7. V. W. Leontieff, «Letter to the Editor», Science, 9 (July 1982). 
Côté Clio, c'est une période de flirt éhonté et d'infidélités. Elle est sollicitée de partout; on la courtise de tous les coins. C'est la multiplication des histoires les plus éclatées. Ce qui fait qu'à mesure qu'Hermès se fait distant, Clio va tomber dans les bras de tous un chacun: histoire des villes, des sciences, des femmes, etc.

Enfin, Hermès, avec l'aide du sorcier américain NEH (New Economic History), va prétendre avoir gain de cause en prenant les grands moyens: on va vouloir fondamentalement Clio - métriser la belle une opération douloureuse et réductrice qui va faire plus de mal que de bien. Ni Hermès ni Clio ne vont plus y trouver leur compte ${ }^{8}$.

Même si la cliométrie a fait moins de ravages au Canada qu'aux États-Unis, les rapports entre Hermès et Clio vont être dramatiquement affectés. Combiné au méthodisme formalisateur, la cliométrisation que voudra imposer l'économie à l'histoire va entraîner la rupture. Les économistes disant s'intéresser à l'histoire vont, pour être acceptés par leurs pairs, s'adonner à des pratiques qui n'ont avec l'histoire que de bien vagues rapports. C'est une période noire de fétichismes du modèle où se développent les pratiques les moins avouables devant les icônes contre-factuels. Les amis de Clio, comme Jacques Barzun, vont alors passer à l'attaque: ils vont définir l'histoire comme anti-abstraction et anti-méthode et briser bien des lances contre le jargon vandalisateur des sciences humaines, et contre les charlatans qui n'auraient pas beaucoup plus à offrir à Clio, concluera Barzun, que l'insémination artificielle ${ }^{9}$. Ces conflits baignés dans l'intolérance n'ont souvent pas permis de voir qu'entre le méthodisme et l'anti-méthode passait le chemin étroit de l'analyse institutionnelle.

Cette période noire a été plus heureuse pour Clio que pour Hermès. Courtisée, entourée, adulée, Hermès n'a pas eu l'air de lui

8. À la suggestion d'un évaluateur anonyme, il semble important ici de nuancer ce qui pourrait sembler un jugement infamant et absolu. Notre malaise tient surtout à l'usage abusif que les épigones de Douglass North ont fait de la cliométrie dans un premier temps. Ces travaux dont Robert Fogel (première manière) sont les meilleurs exemples [R. W. Fogel, Railroads and American Economic Growth (Baltimore, Johns Hopkins, 1964)] proposaient des comparaisons aussi précises que trompeuses. Au Canada, les travaux de G. W. Bertram, E. J. Chambers, John H. Dales, O. J. Firestone, A. G. Green et R. M. MacInnis, entre autres, vont développer ces approches. Pour une énumération de ces travaux, voir T. J. O. Dick, Economic History of Canada (Detroit, Gale Research Company, 1978). Dans les années 1970, Douglass North, Paul David et bien d'autres se sont échappés du carcan cliométrique et ont été parmi les premiers à déborder les impératifs quantophréniques pour s'ouvrir à l'analyse institutionnelle. Voilà qui devait culminer dans la synthèse de D. C. North, Institutions, Institutional Change and Economic Performance (Cambridge, Cambridge University Press, 1990). Cette dérive a commencé dans les années 1970 aux États-Unis mais ne devait donner que peu d'échos au Canada. L'un des produits intermédiaires tributaire en partie de l'une et l'autre époque a été le travail de R. Armstrong, Structure and Change (Toronto, Gage Publishing, 1984).

9. Jacques Barzun, Clio and the Doctors (Chicago, 1974). 
manquer. Mais, après un moment, Clio manque beaucoup à Hermès, même si ce dernier a mis bien du temps à l'admettre, et si dans les cercles économistes purs, on continue à nier que c'est le cas.

En fait, certains cousins européens et américains ont commencé il y a une quinzaine d'années à bourrer leurs lettres à Hermès de toutes sortes de références au charme discret de Clio. En fait, tout un pan de la science économique aux États-Unis et en Europe va redécouvrir l'importance de l'histoire et des institutions ${ }^{10}$.

Ce fort courant institutionnaliste, mettant l'accent sur les formes organisationnelles, s'est développé au cours de la dernière décennie avec énormément de vigueur chez certains groupes d'économistes. Douglass North a même déserté le camp cliométrique pour passer chez les institutionnalistes dans les années 1970, et on a vu un grand nombre d'économistes historiens américains mettre la main au chantier de construction d'un paradigme techno-institutionnaliste ${ }^{11}$. Même si ce n'est pas encore la dominante au Canada et au Québec où, à cause de délais culturels bien connus, la vague méthodiste continue de faire des ravages, un intérêt pour l'histoire économique institutionnaliste a commencé de germer ${ }^{12}$.

Voilà d'ailleurs pourquoi même si certains donnent pour définitivement morte la liaison entre Clio et Hermès, ce constat semble prématuré. En effet, la rumeur veut que Clio et Hermès aient été aperçus dans certains terrains vagues au pourtour de l'académie (dans les écoles d'administration et d'ingénierie): des lieux où les études d'organisation ont pignon sur rue, et où une tradition d'économie historique à la Alfred Chandler ${ }^{13}$ n'a jamais eu de mal à survivre. Clio et Hermès ont eu l'occasion de s'y re-rencontrer au Canada et au Québec, et une histoire économique assez différente pourrait donc y pousser comme des fleurs nouvelles.

À savoir quelles sortes de travaux pourraient en sortir, mieux vaut, avant de spéculer, examiner d'un peu plus près le caractère d'Hermès et de Clio pour jauger les possibles et les probables, pour comprendre aussi ce que pourrait bien vouloir dire cette interaction symbiotique nouvelle dont l'aruspice européen Robert Boyer a parlé ${ }^{14}$. 1990).

10. Pour une revue de la littérature, Claude Ménard, L'économie des organisations (Paris,

11. On a fait une présentation simple de plusieurs dimensions importantes de ce paradigme dans W. Parker, ed., Economic History and the Modern Economist (Oxford, Blackwell, 1986) et dans G. Dosi et al., ed., Technical Change and Economic Theory (London, 1988).

12. R. Armstrong, op. cit.

13. Alfred D. Chandler, Structure and Strategy (Cambridge, 1962); The Visible Hand (Cambridge, 1977).

14. Robert Boyer, «Économie et histoire: vers de nouvelles alliances», Annales. ESC (novembre-décembre 1989): 1397-1426. 


\section{3 - ANALYSE CARACTÉRIELLE}

Clio et Hermès occupent au plan épistémologique des positions antagonistes: comme l'expliquait Fernand Dumont, pour l'histoire, l'univers scientifique procède de l'univers des valeurs, alors que pour la théorie économique, l'univers des valeurs procède de l'univers scientifique ${ }^{15}$.

L'histoire économique et l'économie historique ne seront donc pas produit de la même farine: la loyauté première de l'économiste est à son modèle, c'est-à-dire à une manière de voir; celle de l'historien à l'ensemble des phénomènes qu'il veut comprendre et interpréter. Il va en sortir une élégance, une rigueur et souvent une certaine rigidité de la démarche du premier, alors que la démarche du second sera plus floue et nécessairement éclectique. Ces démarches sont complémentaires en un sens et peuvent être dialectiquement imbriquées, mais elles visent à des constructions de l'esprit, à un certain bricolage dans les deux cas. Pour qu'il y ait coopération cependant, il faut que chacun connaisse bien les pouvoirs et les limites de l'outillage mental du partenaire, sans être intimidé.

Les économistes ont une telle superbe qu'ils ne sont pas en occasion prochaine de péché de modestie. Ils seraient plutôt tentés de considérer les historiens et les archivistes comme des manœuvres dans un chantier dont eux seraient les contremaîtres. Ils se sont donc retranchés dans leurs propres terroirs et se sont déclarés maîtres des lieux en proposant un certain nombre de rationalisations, de schémas incroyablement simplificateurs.

On trouve un bel exemple de ce réductionnisme de l'approche économistique dans certains travaux sur le comportement des autochtones dans le commerce des fourrures. John McManus donne une explication du fait qu'il y a chasse excessive. C'est le cas, dit-il, même quand des droits de propriété privée sur les lieux de chasse ont été mis en place et devraient éliminer une sur-chasse qui est courante quand la ressource est en propriété collective. McManus attribue cette déprédation excessive à un phénomène de bon samaritain: une sorte de police d'assurance primitive par laquelle une communauté aurait toujours droit de chasse dans le territoire d'autrui quand c'est pour assurer sa survie. Voilà qui aurait pour effet d'entraîner des communautés qui maximisent le gibier recueilli par la chasse, sous la contrainte d'une préservation des stocks de ressources à long terme, à

15. Fernand Dumont, «La référence aux valeurs dans les sciences de l'homme», Anthropologica, NS, 1,1-2 (1959). 
tolérer des excès de chasse et donc, à plus long terme, une réduction des stocks de ressources à cause du «comportement de bon samaritain».

Si on insère ce genre d'explication dans le contexte de sociétés autochtones dont la dégradation et la dissolution progressive sont déjà fort avancées, ces ajustements marginaux «au nom du bon samaritain» deviennent des épiphénomènes qui ne sauraient jouer qu'un rôle mineur dans la définition des stocks de ressources. On voit donc que l'explication McManus n'en est pas une. C'est tout au plus une rationalisation. En contraste, l'interprétation suggérée par Abraham Rotstein qui insère le prix des fourrures dans son «contexte» (i.e. un réseau d'arrangements socio-politiques) est à la fois plus complexe mais aussi plus convaincante ${ }^{16}$.

Comme le suggèrent les critiques de la science économique, Albert Hirschman et Fred Block par exemple, en «contextualisant», on complexifie, mais ce qui marque justement les analyses économiques c'est l'enfermement qu'elles produisent et leur trop grande parcimonie $^{17}$.

Cette mise en garde par rapport à Hermès pourra sembler déplacée de la part d'un économiste. Mais il suffira de rappeler qu'Hermès débuta comme voleur de génisses; qu'il s'est auto-proclamé dieu; et que quand Zeus lui confia le portefeuille des contrats, du commerce et de la libre circulation des voyageurs, Hermès promit à Zeus de ne pas mentir, mais ne s'engagea jamais à dire toute la vérité.

Tout cela ne veut pas dire que Clio soit pour autant exempte de travers: elle insiste pour avoir des clartés de tout, une vision totalisante et globalisante. Comme en musique, cette image se développe comme des variations sur un thème qui devient l'élément intégrateur ainsi que le suggérera Donald Creighton. En ce sens-là, Clio est une artiste qui raconte une histoire et ambitionne de saisir la totalité dans sa fresque. Son anti-méthodisme et la définition de l'histoire comme des variations sur un thème - que l'historien choisit arbitrairement et déclare être le thème qui soustend le matériel historique - débouchent sur un grand relativisme puisque ce sont les valeurs de l'historien qui, en dernière instance, déterminent à la fois l'approche à suivre et les critères par lesquels on peut déterminer que la vision totalisante a été réalisée ${ }^{18}$.

16. John C. McManus, «An Economic Analysis of Indian Behavior in the North American Fur Trade», Journal of Economic History, 32 (1972): 78-91; Abraham Rotstein, The Precarious Homestead (Toronto, 1973), chapitre 10.

17. Fred Block, Postindustrial Possibilities (Berkeley, 1990); Albert Hirschman, «Against Parsimony», Economics and Philosophy, 1 (1985): 7-21.

18. Donald Creighton, Towards the Discovery of Canada (Toronto, 1972). 
C'est au plan des méthodes de travail donc, et, puisqu'il faut l'appeler par son nom, de l'épistémologie, qu'on peut cerner le mieux les incompatibilités de caractères d'Hermès et de Clio. Ainsi que le souligne fort bien un ami de la famille, Lucien Goldmann, Hermès a une préférence marquée pour la structure, alors que Clio est plutôt portée sur la phénoménologie: alors que Clio privilégie les faits de conscience, Hermès y substitue un nouvel intelligible construit qu'il déclare être l'essence du réel ${ }^{19}$.

Roland Barthes a bien su décoder l'activité structuraliste d'Hermès qui va produire un simulacre construit du réel, et va supposément, ce faisant, faire «apparaître quelque chose qui restait invisible, ou si l'on préfère inintelligible dans l'objet naturel»; cette anamorphose, cette «transformation surveillée» est réductrice et contingente: elle ne constitue qu'une des mises en forme rationnelle possibles de l'expérience, mais comme le dira bien Fernand Braudel, cette transformation va construire une route à travers nos connaissances incertaines et ne permettre «qu'un seul voyage mais privilégié». Hermès ne ment pas, il dit une vérité partielle et partiale ${ }^{20}$.

Ces contrastes - entre le goût pour le grand voyage et celui pour le sentier privilégié, entre la priorité à la sémantique plutôt qu'à la syntaxe, entre le dominium de la littérature et celui de la science sont au cœur des conflits caractériels qui grippent les rapports entre Clio et Hermès. Or ces traits caractériels sont assez profondément inscrits dans la persona de chacun qu'il est bien improbable que l'un et l'autre changent beaucoup, tout au moins tant qu'ils demeureront dans le sein de leurs tribus respectives.

\section{4 - LE NOUVEAU PARADIGME}

La double crise de l'économie et de l'histoire dans les années 1970-1980 - la première achoppant dans ses explications de la crise, la seconde éclatant en mille morceaux sous le coup des incursions de toutes sortes de disciplines - va faire qu'à la fin des années 1980 de nouvelles alliances ont l'air de commencer à vouloir se forger entre Hermès et Clio, et que le dialogue impossible il y a vingt ans est, diton, en train de reprendre.

L'économie industrielle et managériale a commencé à sentir le besoin de réintroduire le temps irréversible de l'histoire et la trame des

19. Lucien Goldmann, Sciences humaines et philosophie (Paris, 1966).

20. Roland Barthes, «L'activité structuraliste», Les lettres nouvelles, 32 (1963); Roland Barthes, Critique et vérité (Paris, 1966); Fernand Braudel, «Pour une histoire sérielle», Annales ESC, 18 (1963): 541-553. 
institutions dans ses constructions. On a commencé une dérive de l'économie (lente et contestée mais irrécusable) de modèles à saveur scientiste en temps mécanique et réversible, qui veulent expliquer, vers une économie néo-institutionnaliste en temps historique qui cherche surtout à comprendre et interpréter. Le petit livre-manifeste sous la direction de William Parker en 1986 a annoncé le virage, et certains travaux, des deux côtés de l'Atlantique, ont commencé à montrer la voie. Au centre de ces volontés de décloisonnement, il y a un effort pour mieux prendre en compte les dimensions technologiques et organisationnelles ${ }^{21}$. Au Canada, les travaux de Richard Lipsey - un grand converti - promettent $^{22}$.

L'histoire n'a pas pardonné les insuccès passés des modèles simplistes et mécaniques colportés par les économistes. Ce qui fait que malgré les invitations, elle hésite encore à se lancer dans la recherche (pourtant essentielle) de problématiques souples qui puissent guider sans contraindre, de peur de retomber dans les mêmes ornières scientistes du passé. L.es historiens les plus jeunes sont moins hésitants à se risquer sur le terrain miné des économistes, mais, chat échaudé craint l'eau froide.

De plus il y a des empêchements à ces nouvelles fréquentations: Hermès et Clio ont bien changé entre-temps et ont maintenant une certaine difficulté à communiquer. L'orthodoxie économistique continue à produire des schémas simplificateurs, mais dans un langage de plus en plus hermétique. La formation des historiens les prépare mal à décoder ces constructions et les rend souvent incapables de débronzer les fumisteries économistiques à saveur technique et à les départager des modélisations utiles. Les historiens doivent donc travailler dans le maquis à réinventer une économie utilisable, à leur mesure, souvent avec des moyens souvent artisanaux.

Un nouveau paradigme pluriel et souple est pourtant déjà vaguement visible. Nous en avons proposé une première radiographie appliquée au Canada dans un texte de 1982 publié dans cette revue et écrit en collaboration avec Jean-Pierre Wallot ${ }^{23}$. Cette stratégie méso-

21. William Parker, ed., Economic History and the Modern Economist (Oxford, 1986); voir aussi dans un registre plus ample Jacques Lesourne, Économie de l'ordre et du désordre (Paris, 1991).

22. Richard Lipsey, Economic Growth: Science and Technology and Institutional Change in a Global Economy (Toronto, Canadian Institute for Advanced Research, 1991).

23. Cette problématique part de la notion de socio-économie en tant que processus institué et en examine la trame institutionnelle/organisationnelle (Monde 3 de Popper) en tant que faisceau constitutif d'une constellation d'armistices entre faits de conscience (Monde 2 de Popper) et faits matériels (Monde 1 de Popper), entre valeurs et plans d'une part et contraintes 
analytique (examen de la dynamique du processus évolutif de la socioéconomie via l'étude d'un certain nombre de sous-procès) n'est qu'une des stratégies possibles. Mais toutes les stratégies en vogue (venues de terroirs les plus divers) ont un certain nombre de caractéristiques en commun:

- le temps est irréversible et le développement des socio-économies perçu comme se faisant le long de trajectoires plus ou moins différentes;

- la trame organisationnelle de l'économie ainsi que la genèse et l'évolution des institutions sont au centre des analyses;

- la technologie n'est plus exogène mais est une dimension cruciale de la socio-économie comme système informationnel et cognitif en évolution;

- les contextes socio-politiques et culturels doivent nécessairement être pris en compte;

- incertitude, complexité, rationalité limitée et opportunisme sont omniprésents;

- l'auto-organisation (i.e. la possibilité pour un système d'acquérir des propriétés nouvelles en s'organisant ou en modifiant lui-même son organisation) est un processus central.

Ces six éléments conforment une problématique assez différente de celle que colporte la science économique néo-classique. Celle-ci postule que la sphère économique est complètement autonomisée par rapport au reste de la société et que les individus y font des choix rationnels de maximisation de leur «ophélimité» (utilité) sous la double contrainte de préférences et de technologies données. Pas d'apprentissage et pas d'évolution. De plus les modèles d'équilibre général des économistes néo-classiques opèrent dans un temps purement symétrique et mécanique et fonctionnent dans un cadre institutionnel donné.

En encastrant la problématique économique dans son cadre social et culturel, en mettant l'accent sur la genèse des institutions en tant que conventions sociales, en reconnaissant que la socio-économie est un système cognitif en apprentissage qui produit les nouvelles technologies tant physiques que sociales, et en libérant l'individu du carcan d'un rationalisme étroit, une problématique émerge qui est susceptible d'éclairer la démarche de l'historien sans pourtant trop la contraindre.

géo-techniques d'autre part. Gilles Paquet et Jean-Pierre Wallot, «Sur quelques discontinuités dans l'expérience socio-économique du Québec: une hypothèse», Revue d'histoire de l'Amérique française, 35,4 (mars 1982): 483-521; Karl Popper [1972], Objective Knowledge (London, Oxford University Press), chapitre 3. 
$\mathrm{Au}$ lieu de mettre l'accent exclusivement sur une perspective structuraliste et synchronique (i.e. atemporelle) où, comme dirait Frédéric Mauro, «les systèmes se définissent par une certaine imbrication des structures et les structures par un certain nombre de mécanismes type ${ }^{24}{ }^{»}$, on reconnait que l'introduction du temps et de la diachronie réintroduit les événements, les processus, le fonctionnement. Dans ce nouveau contexte, l'intercréation entre structure et fonctionnement, la tension entre synchronie et diachronie, et la démarche récurrente entre ces deux dimensions peuvent articuler et confronter structure et événement, et établir entre les deux des correspondances qui ne soient plus arbitraires. Ce qui sort de cette problématique, c'est «a type of economic theory that deals with long-run, discontinuous, irreversible change over time», comme la nommera Hugh Aitken ${ }^{25}$.

\section{5 - DEUX ALLÉES PROMETTEUSES}

Entre Hermès et Clio cependant commencent à se glisser des entremetteurs. Deux groupes en particulier ont suggéré des formes d'accommodements. Mais trois conditions sont nécessaires pour une union durable entre des partenaires aussi difficiles: 1 - la reconnaissance explicite d'une multiplicité de logiques; 2 - l'accord sur un outillage mental fait de notions intermédiaires entre Hermès et Clio, et; 3 - un pouvoir heuristique évident de cette relation pour les deux partenaires.

A - Les gens des écoles d'administration comme Chandler aux États-Unis ont un pied dans chaque terroir et ont redonné, via des travaux d'économistes comme Oliver Williamson, par exemple, le goût de l'histoire parce que pour étudier le changement organisationnel seules les études diachroniques permettent de sonder la dynamique sous-jacente ${ }^{26}$.

Ces gens des écoles d'administration ont aussi développé des perspectives plus englobantes et automatiquement pluri-disciplinaires. Ils ont construit ainsi des problématiques fort différentes de celles qui

24. Frédéric Mauro [1959], «Théorie économique et histoire économique», Cahiers de l'Institut de Science économique Appliquée, 79, M-4, 45.

25. Hugh G. J. Aitken [1960], «On the Present State of Economic History», Canadian Journal of Economics and Political Science, 26,1 (February): 87-95.

26. Oliver Williamson, Market and Hierarchies (New York, 1975); The Economic Institutions of Capitalism (New York, 1985); pour un examen de la littérature sur les réseaux qui constitue la contribution majeure à la littérature post-Williamson sur les réseaux comme forme organisationnelle hybride entre la firme et le marché, voir Albert Bressand, Catherine Distler et Kalypso Nicolaïdis, "Vers une économie de réseaux», Politique industrielle (Hiver 1989): 155168. 
ont cours dans les départements de science économique: leurs cadres d'analyse ont eu le mérite de démontrer clairement leur utilité dans l'analyse des stratégies des firmes et des politiques publiques, car ces travaux ont mis l'accent sur le temps irréversible, ont placé les institutions au centre de l'économie, n'ont pas accepté de bannir la technologie au rang des choses postulées (parce que justement les choix technologiques sont tout autant que les choix organisationnels ou les choix de localisation, au cœur des stratégies des firmes), et enfin n'ont pas accepté de se confiner à l'examen des comportements de maximisation de la richesse parce qu'il était clair que le comportement dans l'organisation est souvent commandé par des forces d'un autre ordre que celui du rationalisme calculateur.

Pour ces études techno-économiques, la devise est toujours: les choses doivent être contextualisées si l'on veut comprendre les stratégies et débusquer les forces qui gèrent le processus d'autoréorganisation de la socio-économie ${ }^{27}$.

B - Un second groupe d'experts, dont les travaux sont assez bien illustrés par Giovanni Dosi et ses collègues dans leur livre de 1988, ont cherché à prendre en compte directement et centralement la technologie dans l'analyse des socio-économies, et ont été entraînés à mettre au centre du tapis des notions nouvelles comme celles de trajectoires et de filières technologiques et à redéfinir la notion de productivité selon des canons schumpéteriens ${ }^{28}$.

Ces travaux, à l'interface de l'économie de l'histoire et de l'ingénierie, et construits sur les notions de discontinuités, irréversibilités, interactions entre politique, social et économique, ont redonné à la démarche de l'histoire économique une certaine centralité parce que c'est celle qui permettait d'explorer certains cheminements critiques et de comprendre les mécanismes qui ont conduit à certains choix technologiques inefficaces mais pourtant irréversibles (comme par exemple le choix de la configuration du clavier QWERTY ${ }^{29}$. On sait

27. Cette perspective que Richard Lipsey nomme «the Historical, Business School View» correspond en fait exactement à ce que Fred Block nomme «economic sociology» et à ce que recherche Jacques Lesourne dans ses travaux sur l'auto-organisation. Dans chaque cas, le problème central est la genèse des institutions et le caractère fondamental de la socio-économie comme processus institué au sens de Polanyi (Karl Polanyi, «The Economy as Instituted Process «, K. Polanyi, C. M. Arensberg, H. W. Pearson, eds., Trade and Markets in the Early Empires (New York, 1957), 243-270.

28. Pour un examen de la notion d'efficacité schumpéterienne à long terme, voir Giovanni Dosi et al., ed., Technical Change and Economic Theory (London, 1988), ou Giovanni Dosi, John Zysman et Laura D'Andrea Tyson, «Technology, Trade Policy and Schumpeterian Efficiencies», John De la Mothe et Louis Marc Ducharme, dir., Science, Technology and Free Trade (London, 1990), chapitre 3.

29. Voir l'article de Paul David dans William Parker op. cit. 
la complexité des débats contemporains autour de certaines normes et standards en électronique qui sont en train de se vivre, et les enseignements que l'analyse de certaines dynamiques passées ont pu apporter pour éclairer les scénarios probables.

Ces travaux qui ont été longtemps relayés à la périphérie de la science économique sont en train de prendre un ascendant extraordinaire dans les études récentes sur la croisssance économique. Ils sont par exemple au centre des travaux de l'équipe que Richard Lipsey a réunie autour de lui pour le Canadian Institute for Advanced Research. Ces travaux à saveur évolutionnaire, axés sur la politique scientifique et technologique en bonne partie, ont forgé leur propre vocabulaire à l'interface des diverses sciences humaines et de l'histoire ${ }^{30}$.

Dans ces deux avenues ouvertes par les gens des écoles d'administration et par les spécialistes de la technologie, on voit que les présomptions de l'économie néo-classique sont renversées à 180 degrés et que les six caractéristiques mentionnées à la dernière section sont présentes. Ces deux avenues permettent aussi la coexistence de logiques multiples, semblent construire sur un outillage mental intermédiaire, et avoir un pouvoir heuristique évident pour les deux partenaires $^{31}$.

\section{6 - CONCLUSION}

Ces deux avenues nouvelles ont la possibilité de réhabiliter l'histoire économique et de réconcilier les projets de Clio et d'Hermès. Cependant, dans l'un et l'autre cas, les problématiques ne sont encore bien campées et l'outillage mental pas suffisamment bien défini. Ce qui plus est, dans l'un et l'autre terroir, Clio est nettement inféodée à Hermès par les lois du milieu. Le danger est donc grand que Clio, après un moment d'euphorie, ne s'y sente à l'étroit.

Dans un monde où la formation de l'économiste est devenue extrêmement sophistiquée et pointue, et où l'histoire a des ambitions de plus en plus globalisantes, c'est au niveau méso-analytique (des «middle range phenomena») que le travail le plus utile pourra se faire. Pour construire des rapports renouvelés entre Hermès et Clio, il faudra que l'économie ouvre un peu ses perspectives et que l'histoire se fasse

30. Pour une version stylisée de ces travaux en langage économique conventionnel, voir Giovanni Dosi, Keith Pavitt et Luc Soete, The Economics of Technical Change and International Trade (London, 1990).

31. Ce sont des affirmations qu'on pourrait facilement valider et qui transparaissent à la lecture de Dosi et al. et dans toute la littérature post-Williamson sur les réseaux. 
plus modeste. En histoire économique, tout ce qu'on peut espérer c'est mettre de l'avant des «modest general propositions ${ }^{32}$ ».

Malgré le calme des sous-bois pour le moment, l'âge d'or de l'économie historique et de l'histoire économique n'est pas pour demain. Chez nous, le scientisme a commencé d'envahir les écoles de gestion et d'ingénierie et à marginaliser les travaux d'histoire économique et technologique, ce qui fait que la lune de miel pourrait durer assez peu longtemps. Clio et Hermès pourraient bien n'avoir trouvé un havre de paix que pour un petit moment. En fait à les écouter parler, il est clair qu'ils sont prêts à ré-émigrer, et les centres de recherche pluri-disciplinaires à la marge des départements disciplinaires ont commencé aussi à donner asile temporairement à notre couple d'amis.

Quand l'environnement est turbulent, des formes d'accommodements entre des organisations ou des êtres dont les destinées sont, d'une certaine façon, antagonistes deviennent possibles. Les optimistes espèrent que les nouvelles alliances d'Hermès et de Clio vont porter de si beaux fruits dans ces landes nouvelles qu'ils pourront y fonder un foyer permanent. Quant à moi, sans être absolument pessimiste, $\mathrm{j}$ 'avoue envisager comme bien plus probable la possibilité que les historiens économistes et économistes historiens - cette espèce hybride exposée à l'opprobre dont parlait Albert Faucher - soient, comme l'ornithorynque, des espèces en voie de disparition. Il faut admettre cependant que c'est probablement davantage vrai pour le Québec que pour le Canada, et pour le Canada que pour les États-Unis ou l'Europe, pour des raisons qui réclameraient des développements suffisamment importants pour qu'on soit obligé d'en faire l'économie dans un texte comme celui-ci.

32. Arthur H. Cole, «Meso-Economics: a Contribution from Entrepreneurial History», Explorations in Entrepreneurial History, 2nd Series, 6,1 (1968): 3-33; Gilles Paquet et JeanPierre Wallot, op. cit.; Gilles Paquet, "Capital Cities as Symbolic Resources», Les capitales: conditions de leur développement efficace et harmonieux (Ottawa, 1993) (à paraitre). 